\title{
Citation:
}

Article No.: 1339

Szymański, T. (2017). Les cours parisiens d’Adam Mickiewicz et l'idée de religion universelle. Slavia Meridionalis, 17. https:// doi.org/10.11649/sm.1339

\section{Tomasz Szymański}

Uniwersytet Wrocławski

\section{Les cours parisiens d'Adam Mickiewicz et l'idée de religion universelle}

Les cours parisiens d'Adam Mickiewicz sur La littérature slave, donnés au Collège de France de décembre 1840 à mai 1844, et dont l'importance a été soulignée par les plus grands mickiewiczologues ${ }^{1}$ (Stefanowska, 1976, 2014; Piwińska, 1997; Weintraub, 1975, 1998a, 1998b), ont toujours constitué une matière difficile pour les chercheurs, posant des problèmes de réception et d'interprétation innombrables (Kuziak, 2007; Kubik, 2012). Les spécialistes en littérature - malgré le fait que les cours doivent être traités comme une œuvre littéraire, et peut-être même avant tout littéraire - peuvent être découragés par leur orientation philosophico-religieuse, leur dimension prophétique réduisant la poésie à la transmission d'un message religieux spiritualiste.

${ }^{1}$ Les cours ont été notamment l'objet du volume offert à Zofia Stefanowska, dirigé par Maria Kalinowska, Jarosław Ławski et Magdalena Bizior-Dombrowska (Kalinowska, Ławski, \& Bizior-Dombrowska, 2011).

This work was supported by the Polish Ministry of Science and Higher Education.

Competing interests: no competing interests have been declared.

Publisher: Institute of Slavic Studies, Polish Academy of Sciences.

This is an Open Access article distributed under the terms of the Creative Commons Attribution 3.0 PL License (creativecommons.org/licenses/by/3.0/pl/), which permits redistribution, commercial and non-commercial, provided that the article is properly cited. (c) The Author(s) 2017. 
Les philosophes, eux, se trouvent déconcertés par la tonalité radicalement antirationaliste et antiphilosophique des cours. Leur étude n'est pas facilitée par l'état d'édition actuel des textes ${ }^{2}$, édition tiraillée entre la parole vivante que les sténographes ont tenté de noter, et les versions remaniées et publiées en français aussi bien que dans d'autres langues, suivant des critères divergents, parfois confus (Weintraub, 1998a; Prussak, 2011). On a toutefois attiré l'attention sur la valeur des cours du point de vue de l'histoire des idées - s'y entrecroisent en effet de nombreuses influences littéraires, religieuses, philosophiques et anthropologiques, et leur rencontre peut jeter une nouvelle lumière sur le contexte et l'atmosphère dans lesquels les cours se sont tenus, qui sont ceux de la période troublée de la Monarchie de Juillet ${ }^{3}$.

Des spécialistes de Mickiewicz comme Andrzej Walicki ou Wiktor Weintraub ont démontré l'ancrage profond des cours de Mickiewicz dans la culture européenne et particulièrement française. Les émigrés venant d'une Pologne partagée qui a perdu son indépendance viennent puiser leur inspiration dans les mouvements traversant les pays qui les accueillent, à commencer par la France. Le messianisme polonais doit être mis en rapport avec les doctrines des illuminés, des millénaristes, des utopistes et des socialistes romantiques, qui se développent dans la première moitié du XIX ${ }^{\mathrm{e}}$ siècle (Walicki, 2002, 2006, 2009). Mais en même temps, les Polonais apportent leur propres idées et intuitions, qui associées aux influences mentionnées, donnent en résultat un alliage culturel intéressant, parfois singulier, où on essaye de fournir des réponses aux questions pressantes de l'époque : oppression des peuples, paupérisation, effondrement des anciennes valeurs et lutte pour les valeurs émergentes. Dans cette recherche de nouvelles solutions politiques, sociales et religieuses, la formulation de l'idée d'une religion universelle, d'une nouvelle religion ou d'une religion de l'avenir joue un rôle de premier plan. C'est dans cette perspective là que nous voulons nous pencher sur les cours mickiewicziens.

La religion a toujours joué un rôle socio-politique déterminant dans le monde occidental. Le christianisme y a servi de fondement et de ciment social à partir du moment où il a été déclaré religion impériale, et n’a pas perdu son statut jusqu’à

${ }^{2}$ Nous ne disposons toujours pas d'une édition critique complète des cours en français. La traduction en polonais de Léon Płoszewski qui fait actuellement autorité constitue une synthèse hétéroclite entre les sténogrammes et les éditions publiées en français en 1845 et 1849 (qui diffèrent considérablement des premiers).

${ }^{3}$ Pour les détails historico-biographiques de la période des cours, voir la chronique de la vie et de l'œuvre de Mickiewicz de Zofia Makowiecka (1968). 
l'éclatement de «la crise de la conscience européenne» (Hazard, 1935) à l'aube des Lumières. Jadis attitude de piété et à partir des temps modernes - culte fondé sur une doctrine particulière, la religion est alors soumise à une critique permanente qui aboutira à des réinterprétations radicales de ses composantes rituelles, doctrinales, communautaires et morales. La réflexion moderne sur la pluralité des religions mène au dépassement des cultes particuliers dans différents sens (tantôt rationalistes, tantôt mystiques) et à la recherche de leur dénominateur commun, donnant ainsi naissance à de nombreuses variantes de la religion universelle : celle des apologistes catholiques, celle des illuminés - issue d'une tradition ésotérique, celle des philosophes - rationaliste et naturelle, celles des franc-maçons - associant révélation et libre-pensée, ou encore celle des naturalistes radicaux, qui refusent à la religion toute raison d'être. Ces différentes conceptions de la religion universelle vont trouver leurs prolongements au XIX ${ }^{\mathrm{e}}$ siècle, qui les enrichit de nouveaux contextes : socialisme romantique et utopique, positivisme, ultramontanisme ou humanitarisme laïc. C'est à la lumière du croisement de ces tendances qu'il convient de se pencher sur l'œuvre de Mickiewicz et des ses cours au Collège de France. Comme nous l'avons indiqué, des chercheurs comme Walicki, Weintraub ou Michel Masłowski $(1998,2002)$ ont déjà consacré des travaux aux interférences entre les cours et les idées religieuses de l'époque ${ }^{4}$, issues des milieux philosophiques français et allemands, ainsi que des courants ésotériques (particulièrement du martinisme $)^{5}$, millénaristes et utopistes. Cependant, nous voudrions tenter de saisir plus en détail les rapports entre la religion slave trouvant chez Mickiewicz son accomplissement dans le messianisme, et les diverses formes de religion universelle ${ }^{6}$ présentes à l'époque chez de Maistre, Ballanche, Lamennais ou

${ }^{4}$ Il faut aussi mentionner ici l'ouvrage collectif dirigé par Andrzej Fabianowski et Ewa Hoffmann-Piotrowska (Fabianowski \& Hoffmann-Piotrowska, 2005), ayant pour sujet la dimension mystique de l'œuvre de Mickiewicz.

${ }^{5}$ Nous renvoyons aussi à l'essai de Czesław Miłosz La Terre d'Ulro, où l'auteur se penche sur les sources mystiques et ésotériques de Mickiewicz (Bœhme, Saint-Martin, Swedenborg, Blake), notamment dans le contexte de la crise romantique et du «déshéritage de l'esprit » qu'elle a entraîné, décisif pour l'itinéraire littéraire et spirituel de Miłosz.

${ }^{6}$ La question a déjà été abordée ponctuellement par Léon Kolodziej dans sa synthèse impressionnante de la pensée mickiewiczienne, Adam Mickiewicz : au carrefour des romantismes européens, essai sur la pensée du poète, parue en 1966, notamment dans le chapitre «Religion de Mickiewicz» (Kolodziej, 1966, pp. 353-391). Cependant, indépendamment de la thèse principale de l'ouvrage, qui est discutable, nous considérons que le sujet mérite une étude à part, de ce fait plus claire et plus structurée, prenant pour base les cours du Collège de France. 
Leroux. L'importance des enjeux politiques, sociaux et moraux qui s'entremêlent dans cette poliphonie concernant la question religieuse dans les années $40 \mathrm{du}$ siècle nous paraissent justifier une telle entreprise.

Nous allons nous concentrer principalement sur les cours III et IV de Mickiewicz sur la littérature slave, qui ont lieu en 1843 et 1844, et qui sont publiés en 1845 en deux volumes sous les titres respectifs : L'Église officielle et le messianisme et L'Église et le Messie, et portant les sous-titres Littérature et philosophie pour le premier, Religion et politique pour le second tome. Mais étant donné que la question religieuse et celle du messianisme sont aussi traitées, dans une moindre mesure, dans les cours I et II, nous y ferons aussi référence de façon occasionnelle. Nous avons divisé notre étude en deux parties : la première traitant de la tentative mickiewiczienne d'accéder à une révélation primitive antérieure à la révélation juive et chrétienne, et la deuxième consacrée à l'annonce d'une révélation ultérieure à cette dernière, qui vient la compléter et la parfaire.

\section{La révélation avant la révélation}

La première question qui s'impose lorsqu'on aborde le problème qui nous intéresse est celui de la révélation. Il fournit en effet le principal critère de différenciation concernant l'idée de religion universelle : les temps modernes ont élaboré l'idée de la religion naturelle opposée à la religion révélée ou indépendante de la révélation, sans toujours exclure cette dernière (Lagrée, 1991). Or, il s'agit d'un problème de premier ordre pour Mickiewicz, qui le traite d'une façon complexe, parfois peut-être même contradictoire. Tout d'abord, selon le poète,

${ }^{7}$ Nous utilisons dans l'article les versions imprimées des cours : publiés, pour ce qui est des cours III et IV, sous les titres cités à Paris «Au comptoir des imprimeurs-unis», en 1845 (Mickiewicz, 1845a, 1845b) et pour ce qui est des cours I et II, chez le même éditeur, sous le titre Les Slaves, en 1849 (Mickiewicz, 1849a). Cette dernière édition, en trois volumes, comprend les 74 leçons des cours I et II, réparties selon l'ordre suivant. Premier volume : cours I, leçons 1-25; deuxième volume : cours I, leçons 26-41 et cours II, leçons 1-9 (numérotés 42-50); troisième volume : cours II, leçons 10-33 (51-74). Pour plus de clarté, nous gardons dans l'article la numérotation originale des cours et des leçons, en indiquant entre crochets la numérotation de l'édition de 1849, d'après le modèle suivant: [numéro du volume, leçon, page(s)]. Dans le cas des cours III et IV, nous renvoyons à la numérotation originale: numéro du cours, leçon, page(s). 
la religion slave dans son principe est une religion qui ne connaît pas de révélation. Celle-ci établissant des rapports entre l'homme et le divin, exprimés dans des récits mythologiques, les Slaves ne possèdent à l'origine aucune mythologie, et par conséquent - pas de sacerdoce, ni de hiérarchie, ni d'esprit de conquête. Ce que l'on appelle la «mythologie slave» n'est autre que la relgion slave, et tout élément mythique au sein de cette religion provient d'autres traditions païennes (gréco-romaines ou barbares) et constitue un apport étranger. Car les mythologies anciennes sont en rapport avec la révélation judéo-chrétienne dans la mesure où elles l'on déformée et corrompue. Cependant, d'un autre côté, la religion slave repose sur quelques dogmes principaux : l'existence de Dieu et de son adversaire, ainsi que l'immortalité de l'âme ${ }^{8}$. Elle connaît aussi une vague tradition du péché et de la nécessité du sacrifice (I, V, [I, V, 65-66]; I, VI, [I, VI, 67-70]). Dans le troisième cours, Mickiewicz mentionne aussi le dogme de «l'influence continuelle du monde invisible sur le monde visible», «sur lequel reposait la nationalité slave en général et la nationalité polonaise particulièrement» (III, X, 216; Mickiewicz, 1845a, p. 216).

Il s'agit là d'une ambivalence curieuse, indiquée déjà par Tadeusz Linkner (2014) : la « religion primitive » slave n'est pas révélée, mais semble détenir certaines connaissances provenant d'une révélation, en véhiculer certaines traces. Comment en effet comprendre la présence chez les Slaves des « dogmes» sur l'unité de Dieu et l'immortalité de l'âme, et d'une certaine notion de la chute et de la rédemption? D’autres questions problématiques peuvent être avancées ici : les Grecs, les Romains, les Celtes ou les Germains connaissaient-ils la révélation pour pouvoir la déformer dans leurs mythes ? Selon Mickiewicz, les Slaves, ignorant les sciences et les arts, ayant conservé leur pureté originelle, étaient le mieux disposés à accueillir le christianisme. Ils n’ont pas gaspillé, corrompu et faussé «l'âme de la mythologie» en créations artistiques, poésie et commentaires, et ont réussi à préserver la tradition vivante primtive (III, XII, 233-238). Les quelques dogmes qu'ils avaient adoptés attendaient leur développement et leur explication dans la religion du Christ.

8 Mickiewicz écrit plus haut : «Les Slaves admettaient l'existence d'un Dieu unique ; ils croyaient aussi à l'existence d'un esprit mauvais, d'un dieu noir en lutte avec un dieu blanc, souverain, rémunérateur et vengeur ; enfin ils croyaient à l'immortalité de l'âme. Ces trois dogmes forment toute leur religion [...]» (I, V [I, V, 65]; Mickiewicz, 1849b, p. 65). Zbigniew Kaźmierczyk considère le dualisme d'origine iranienne, caractéristique de la mythologie slave, comme la structure fondatrice de la «psychomachie slave» de Mickiewicz, qui se traduit sur le plan historiosophique dans le conflit polono-russe (Kaźmierczyk, 2012). 
La conception mickiewiczienne de la religion primitive des Slaves évoque à bien des égards la religion des premiers patriarches bibliques, notamment celle de Noé9. Mickiewicz y fait d'ailleurs allusion de façon explicite : «Sa religion [celle du peuple slave] est primitive, patriarchale [sic], telle encore que nous la voyons dans le livre de la Genèse» (I, V [I, V, 65]; Mickiewicz, 1849b, p. 65). Mais il s'agirait alors d'une religion révélée - révélée à l'humanité entière, et antérieure à la révélation mosaïque. Lors de son développement, survenu au sein de la tradition judéo-chrétienne, elle aurait été défigurée par les autres traditions, suivant le principe de l'imitatio diabolica, comme l'enseignaient les Pères de l'Église. La religion primitive des Slaves n'est pas, malgré l'adoption de principes similaires (existence de Dieu, immortalité de l'âme), la religion naturelle des philosophes issue de la réflexion rationnelle, mais plutôt la religion naturelle telle que l'envisage la tradition catholique : anticipation et première étape de la révélation, qu'elle contient déjà en germe. Ce n'est pas non plus, malgré d'autres ressemblances, la "pure religion de l'unité » de Creuzer, que celui-ci expose dans sa Symbolique. Mickiewicz y renvoie dans le troisième cours (III, XII, 223-224), en remarquant que Creuzer, de même que Saint-Martin, ramène les mythologies et les traditions à une source commune. Creuzer associe les manifestations les plus directes de cette source avec les religions orientales. Mickiewicz, d'accord avec des mythologues allemands comme Hanusch, situe les origines du peuple slave et de sa culture en Inde :

Les mythologues sont d'accord pour placer en Asie le berceau de toutes les révélations et de toutes les traditions religieuses. Nork, dans son ouvrage sur les traditions des Syriens, et Hanusch, dans son traité de la mythologie lithuano-slave, acceptent et appuient cette hypothèse. Il s'agit maintenant de déterminer ce qu'il y a, dans la religion des Indous, d'essentiel, de primordial et de propre à cette nation, pour que nous puissions suivre dans les migrations des peuples cet élément indien, et en observer le développement et les transformations. / Le premier dogme de toutes les révélations anciennes est celui de l'unité et de l'éternité de Dieu. Toutes les religions sortent de ce dogme (III, XIII, 240; Mickiewicz, 1845a, p. 240).

Il est intéressant de constater que l'Inde constitue pour Mickiewicz d'un côté « le berceau de toutes les révélations et de toutes les traditions religieuses » (non pas seulement de la tradition slave qui provient directement d'elle), mais

9 La religion de Noé, religion par excellence universelle, est un sujet de prédilection pour des auteurs des temps modernes comme Uriel da Costa, Andrew Michael Ramsay ou Isaac Newton, sous l'influence duquel le noachisme devient le fondement philosophico-religieux de la Grande Loge de Londres, première grande loge maçonnique fondée en 1717. 
de l'autre, la mythologie hindoue ne fait que pressentir la « véritable» révélation. Et contrairement à Nork, il juge la tradition hébraïque supérieure à celle de l'Inde, dont les défauts majeurs sont la passivité contemplative (défaut partagé par la philosophie idéaliste allemande) et l'exclusivisme du système de castes (III, XIII, 248). Pour Mickiewicz, il est hors de doute que le christianisme, précédé du judaïsme, est «la seule religion où la tradition se trouve incarnée » (III, XIII, 255; Mickiewicz, 1845a, p. 255). Mickiewicz pouvait apprécier la méthode intuitive de Creuzer (critiquée par les rationalistes purs), tout en récusant ses liens avec la philosophie idéaliste, ainsi que son caractère contemplatif visant à opérer un retour à l'origine de tous les cultes. En effet, si Mickiewicz rejette, comme Creuzer, le progressisme rationaliste protestant, il n'en adopte pas moins une conception évolutive de la religion et de la révélation. Il ne s'agira donc pas d'effectuer un retour pur et simple à la source, mais de suivre le cours d'une évolution spirituelle, cette évolution supposant parfois des soubresauts révolutionnaires. Mickiewicz, à ce titre, est progressiste. Toute la question est de savoir en quoi réside le moteur du progrès - pour Mickiewicz ce moteur est le Verbe de l'Évangile de Jean, et non le logos des philosophes.

La révélation, en dépit de son absence apparente dans la religion primitive slave, se trouve au centre des préoccupations de Mickiewicz, qui le dit d'ailleurs ouvertement au début du troisième cours, en parlant des bases de la «philosophie » (mot qu'il emploie à contrecœur, faute de pouvoir en trouver un autre) slave:

Cette philosophie accepte d'abord comme une vérité que, dès le commencement, il y avait des sociétés, des dogmes, des débris d'une révélation universelle; que, dans les temps les plus anciens, les hommes possédaient déjà une certaine masse de vérités révélées dont nous ne connaissons pas la source. Plus tard, les hommes qui avaient conservé ce dépôt sacré, et qui avaient fait tous leurs efforts pour l'augmenter et le développer, se sont rendus capables de recevoir une révélation plus haute et plus complète, celle du christianisme (III, I, 8; Mickiewicz, 1845a, p. 8).

L'idée d'une révélation primitive dont procèdent toutes les traditions et toutes les croyances religieuses de l'humanité, révélation qui s'y trouve disséminée et comme obscurcie, a une longue histoire. Adoptée par certains Pères de l'Église et véhiculée par la tradition catholique, l'idée sera constamment réinterprétée en fonction de la manière dont on envisage les rapports entre la tradition originelle et les cultes particuliers. Ainsi, à l'époque de la Renaissance, les grands sages et les mages païens, tels Hermès ou Orphée, trouvent leur place à côté de Moïse parmi les chaînons de la prisca theologia puis de la 
philosophia perennis, tradition transmettant la sagesse de la révélation divine primordiale. Cette conception, parfois associée à l'imaginaire de la tradition maçonnique, sera chère aux théosophes et aux illuminés des temps modernes. Emanuel Swedenborg parle de la transmission de la science des correspondances, connue de la Très-Ancienne Église. Louis-Claude de Saint-Martin évoque les «traditions primitives » et la "Tradition-mère », qui ont leur source dans le savoir et le pouvoir divins du Premier Homme, oubliés mais conservés et transmis par les élus de Dieu. La Parole, principe générateur des êtres, constitue le noyau de la révélation, ou plutôt de l'autorévélation du Premier Principe. L'homme et la création constituent un livre façonné par le magisme de la Parole, et contenant le langage divin, qui n'a rien d'arbitraire, mais renvoie aux sources vivantes et spirituelles de l'être (I, VII [I, VII, 86-87]). Mickiewicz en parle en évoquant la vision poétique de son compatriote Krasiński :

La parole, dit l'auteur de la Comédie infernale, réside dans l'homme, comme Dieu dans l'univers ; elle réside tout entière dans chaque mot, chaque mouvement, chaque action de l'homme, comme Dieu incommensurable existe dans chaque atome, présent partout, et nulle part saisissable. Elle brille, cette parole, comme une étoile fixe sur le front de l'homme ; elle lui donne le vrai caractère d'homme (IV, VIII, 136-137; Mickiewicz, 1845b, pp. 136-137).

Cette idée de la tradition vivante de la Parole ou du Verbe, Mickiewicz l'a vraisemblablement puisée chez Saint-Martin, à l'œuvre duquel il a été initié à Saint-Pétersbourg par le peintre, illuminé et franc-maçon Józef Oleszkiewicz (Weintraub, 1998b). Tout son prophétisme poétique, ainsi que sa critique de l'Église officielle - celle-ci a en effet « complètement perdu l'idée et la tradition de la parole vivante » (IV, VII, 114) - repose sur cette conception martiniste de la Parole divine originelle (Weintraub, 1959). La poésie ne sert finalement qu'à transmettre les vérités supérieures, permettant de jeter la lumière sur le sens de la création et des destinées humaines. Tout comme Saint-Martin, Mickiewicz accorde la primauté sur les autres traditions à la tradition judéo-chrétienne, qui mène à l'œuvre du Réparateur qu'est le Christ. Cependant, la «tradition vivante» dépasse le cadre des révélations juive et chrétienne (conçues de façon étroite) et ouvre la perspective d'une révélation universelle et originelle, qui sera perfectionnée par des « révélations partielles et successives » (III, I, 8; Mickiewicz, 1845a, p. 8). En effet, comme le constate le « wieszcz» polonais :

[...] les versets attribués à Orphée, à Musée, aux sibylles, les sentences des sages, les pensées de Pythagore, portent l'empreinte du spiritualisme le plus pur, et accusent 
un travail de spéculation très avancé. Les Pères de l'Église et les théosophes aimaient à citer les fragments de cette antique sagesse de la Grèce, et y trouvaient des rapports frappants avec les dogmes de la révélation. On voit donc que les instituteurs des peuples primitifs avaient sur la Divinité et sur la création des idées trop hautes pour pouvoir jamais confondre le culte de Dieu avec celui du soleil (III, XIII, 244; Mickiewicz, 1845a, p. 244).

De même que Saint-Martin, Mickiewicz rejette ici les idées de Charles-François Dupuis ou de Volney, qui associent toutes les croyances et mythologies au culte des astres ${ }^{10}$ et leur attribuent une origine purement naturelle. Pour le théosophe aussi bien que pour le poète, la source de la religion est divine et n'est pas de ce monde. Et il n'y a à proprement parler qu'une seule et même religion, dont les dogmes, à commencer par celui de l'existence d'un Dieu unique, peuvent être transmis de façon plus ou moins fidèle, ou bien obscurcis et déformés par l'esprit humain (notamment par les arts, la philosophie, voire la théologie), donnant naissance à des chimères extravagantes ou des systèmes rationalisés stériles. Il existe donc une tradition universelle et vivante - universelle, car présente dans toutes les cultures (quoique de façon privilégiée dans les cultures juive puis chrétienne), et vivante, car ne pouvant être transmise et cultivée que par les gens et les peuples qui la comprennent et l'incorporent dans leur vie:

Ainsi la tradition n'est autre chose que la forme réelle de la vérité, la forme réelle de la vie, tandis que les contes, les récits, les rêveries, les compositions poétiques, ce sont des formes fausses et mensongères, des caricatures de la vérité. La tradition est une vérité transmise par les hommes capables de la sentir et de la pratiquer. Elle suppose l'existence d'un fait avéré et des hommes en état de le reproduire. Il n'y a pas de vérité sans un homme qui la manifeste. Une telle vérité n'a pas d'existence, elle n'a pas de réalité, elle n'a pas commencé à vivre » (III, XIII, 253-254; Mickiewicz, 1845a, pp. 253-254).

Ce n'est que dans ce contexte que l'on peut comprendre pourquoi la mythologie slave, concentrée sur la vie agricole et familiale, et qui se distingue par son sens de la réalité et du devoir (III, XIII, 267-279), renvoie pour Mickiewicz à la «mythologie universelle» et pourquoi «l'intérêt général de la science mythologique se trouve maintenant rattaché à la question slave » (III, XII, 226;

${ }^{10}$ Mickiewicz explique l'universalité du culte solaire et lunaire par le magnétisme et le somnambulisme. Selon lui, les astres exercent une influence sur la communication de l'homme avec la Divinité. Leur culte témoigne de la recherche d'un certain état de clairvoyance (III, XIII, 244-247). 
Mickiewicz, 1845a, p. 226) ${ }^{11}$. Par ailleurs, une place spéciale est accordée par Mickiewicz à la mythologie lituanienne, ayant aussi sa source en Orient, et traduisant la tradition universelle du genre humain dans toute sa plénitude:

Des divinités de diverses hiérarchies visibles ont, dans la langue slave, des noms et des mots qui expliquent leur action et leurs attributs ; mais ce n'est que chez les Lithuaniens qu'on en trouve l'histoire, la filiation, les faits et gestes. Nulle part les idées religieuses n'ont formé un ensemble aussi vaste et aussi complet. Dans les notes fournies par les anciens auteurs de l'Occident qui ont écrit sur ces peuples, et dans la tradition vivante, on a découvert les traces des opinions des brahmanes : sur la nature des âmes humaines, sur l'état de l'âme après la mort, sur les moyens les plus propres à sauver cette âme ; ensuite les traditions de la race zend, de la caste guerrière des adorateurs du feu et de l'eau : sur la lutte des deux principes du feu et de l'eau, sur les devoirs que les enfants du soleil ont à remplir en combattant les générations des ténèbres, et tous les rites qui correspondent à cette religion guerrière ; enfin les rites et les cérémonies consacrés à sanctifier la vie domestique, qui nous rappellent et qui nous expliquent la religion des Grecs et des Romains ; de sorte que nous trouvons dans cette mythologie le brahmanisme des Indes, la tradition grecque et romaine, tous les rites des anciennes idolâtries de l'Europe, et toutes les superstitions de l'Europe moderne (III, XV, 282-283; Mickiewicz, 1845a, pp. 282-283).

L'apologétique catholique, notamment celle du premier quart du XIX ${ }^{\mathrm{e}}$ siècle, revient constamment à l'idée d'une révélation primitive, qui trouve sa manifestation la plus complète dans le catholicisme. Joseph de Maistre, qui comme Saint-Martin a fréquenté les cercles des illuminés et appartenu à la franc-maçonnerie de rite écossais rectifié, ne partage pas avec le théosophe d'Amboise son attitude critique vis-à-vis du clergé catholique. Il adhère pourtant à la conception d'un christianisme transcendant qui rassemble et résume toutes les traditions religieuses. Devenu ultramontain, il associe cette religion aussi ancienne que le monde au catholicisme, qui repose sur l'autorité papale, celle-ci

11 Cette thématique est développée par Mickiewicz dans le deuxième cours, où il traite de la façon dont la religion slave envisage les rapports entre le monde visible et invisible : «Les idées que je viens d'exposer, je les ai puisées dans la tradition populaire slave, conforme à la tradition universelle, et elles ont présidé à la création de nos grandes compositions littéraires » (IV, VI, 96; Mickiewicz, 1845b, p. 96). Et voici un échantillon concret de la «tradition universelle» dont il est question, illustrant la façon dont elle se manifeste: «Ce qui est le plus singulier, c'est que tous les héros et les saints de la sculpture portent pour ainsi dire le même costume, comme s'ils habitaient tous le même pays. On a beaucoup écrit sur ce costume, sur cette draperie, que l'on continue d'appeler dans les écoles le costume grec ou romain. Le fait est qu'il n'est ni grec ni romain. Ce costume est traditionnel, on le sent ; il est dû a une révélation première» (IV, VI, 99-100; Mickiewicz, 1845b, pp. 99-100). 
constituant pour de Maistre le principal critère de vérité - ce que Mickiewicz ne manque pas de relever, ne pouvant d'ailleurs pas se mettre d'accord avec lui sur ce point. En effet, s'il persiste à exprimer son attachement à l'Église romaine, les rapports du poète avec le souverain pontife vont progressivement se détériorer : même s'il passe sous silence les condamnations de l'insurrection de novembre 1830 en Pologne et de son Livre des pélerins polonais ${ }^{12}$, la rupture décisive aura lieu en 1848 (Mickiewicz tente alors d'arracher une bénédiction à Pie IX), rupture préparée bien avant par les foudres lancées contre «l'Église officielle» dans les cours III et IV du Collège de France.

Car Mickiewicz, tout attaché qu'il soit à la tradition catholique, peut passer pour un anticlérical et un partisan de la Révolution, dans laquelle il ne voit pas une conséquence de la philosophie rationaliste des Lumières, mais un passage à l'acte providentiel contre l'injustice et l'oppression, un élan d'enthousiasme inspiré par le Verbe qui pousse l'histoire en avant. C'est ce qui le sépare aussi bien des romantiques et mythologues allemands conservateurs comme Görres ou Friedrich Schlegel, que des traditionalistes français comme de Maistre, Bonald ou le premier Lamennais. Le «catholicisme à la Chateaubriand » ne gagne pas non plus sa sympathie, car il place la forme au-dessus de l'âme et de la tradition vivante, privilégiées par les Slaves (III, IX, 178-181). Pourtant, tous les auteurs cités admettent l'existence d'une tradition universelle, disséminée dans les croyances du monde, véhiculant les vérités sur lesquelles tous les hommes ont toujours été d'accord, et dont le catholicisme offre la traduction la plus parfaite. Ainsi, Lamennais formule sa conception de la "raison générale », opposée à la raison individuelle et critique ${ }^{13}$. Selon lui, il n'est possible d'accéder à la vérité, issue de la révélation donnée à l'homme au commencement, que par une soumission à l'autorité, celle de l'Église et du pape en l'occurrence. Mickiewicz connaît Lamennais et son ouvrage sur L'indifférence en matière de religion, où il expose les idées en question. Dans son troisième cours, il évoque Lamennais comme celui qui place le critère de la certitude dans le « consentement général », dans l'universalité du genre humain :

${ }^{12}$ La traduction du Livre des pélerins polonais par Charles de Montalembert, suivie de l'Hymne à la Pologne de Lamennais, est publiée en 1833.

${ }^{13}$ Lamennais écrit : «Le premier homme reçoit les premières vérités, sur le témoignage de Dieu raison suprême, et elles se conservent parmi les hommes, perpétuellement manifestées par le témoignage universel, expression de la raison générale ( (Lamennais, 1817, p. 96). 
D'après le système de M. Lamennais, le pape n'est pas regardé comme créateur de la vérité, de la certitude, mais comme une espèce de président comptant seulement les votes et constatant les décisions de l'universalité des citoyens qu'il appelle tantôt Église, tantôt chrétienté, tantôt genre humain» (III, XXIII, 453; Mickiewicz, 1845a, p. 453).

Il n'est pas clair à quelle période de la création de Lamennais renvoie la référence, il n'est pas sûr non plus que Mickiewicz ait bien saisi la pensée du Breton. Le premier Lamennais dit plutôt que le pape est le gardien des vérités transmises par la "raison générale», qui ne sont ni discutables ni votables. Et dans le cas où il s'agirait du second Lamennais, démocrate chrétien (sa rupture avec l'Église est déjà consommée à l'époque), la référence au pape perd son sens. À cela s'ajoute le jugement extrêmement défavorable du poète porté sur les Paroles d'un croyant, qui pouvaient être inspirées par Le livre des pélerins polonais (Weintraub, 1998a). Mickiewicz voit dans les Paroles un ouvrage empli de rhétorique, et en Lamennais un polémiste, qui manque d'une véritable inspiration prophétique. Car s'il existe un critère de vérité pour Mickiewicz, c'est précisément le contact intuitif avec la région supérieure, région intérieure et mystérieuse, monde habité par les esprits « où coulent la vie et la vérité », connu de Pythagore et de Platon, et plus récemment, à travers la notion d'intuition, d'Emerson et de Cieszkowski. De ce point de vue là, Mickiewicz, comme on l'a déjà souligné, est fidèle à Saint-Martin, ainsi qu'à Jacob Bœhme, maître à penser du premier, ou encore Angelus Silesius - tous trois ayant alimenté le recueil d'aphorismes mickiewiczien Zdania i uwagi (Notes et pensées) de 1836, d'inspiration à la fois mystique et ésotérique.

Parmi les écrivains français connus de Mickiewicz qui jouent un rôle de premier plan dans la réflexion sur la question religieuse et l'idée d'une religion universelle, il faut encore citer Pierre Leroux. Il est mentionné à côté de de Maistre et de Lamennais lorsque Mickiewicz aborde le problème du critère de la certitude. Pour Leroux, celui-ci ne réside pas dans le «consentement général », mais dans la « raison universelle ${ }^{14}$ :

Ayant médité les religions et l'histoire des peuples, il trouve partout les traces des opinions analogues sur les grandes questions qui divisent l'humanité, sur Dieu, sur l'existence de l'âme et sur les devoirs moraux. Il s'agirait donc de réunir tous les débris et d'en former une opinion qui représenterait celle du genre humain (III, XXIII, 453; Mickiewicz, 1845a, p. 453).

${ }_{14}$ Observation curieuse dans la mesure où c'est Lamennais, précisément, qui utilise le terme de « raison générale». 
Leroux, dans son ouvrage De l'humanité, paru l'année même où Mickiewicz commençe ses cours au Collège de France, tente effectivement de formuler une synthèse religieuse réunissant les anciennes traditions ainsi que la philosophie moderne dans une "révélation éternelle et successive ${ }^{15}$, ce qui n'a sans doute pas échappé à Mickiewicz, pour qui le messianisme est « une série de révélations » (III, II, 20-21). Il appécie le philosophe français pour son idée de "nation-religion » française (II, XXXII, 352), ainsi que pour sa méthode intuitive, objet de critique des «philosophes de l'école» (III, XXIII, 456). Leroux partage avec Emerson, outre cette approche intuitive, l'idée d'une âme universelle absorbant les âmes individuelles, à laquelle Mickiewicz semble adhérer, même si son attitude est ambivalente sur ce point. En effet, il souligne en même temps l'importance dans la tradition slave de la représentation des esprits et des âmes individuelles (IV, VI, 92-94). Et le vampirisme, qui reflète philosophiquement l'individualité de l'esprit humain, constitue la façon poétique privilégiée par laquelle le peuple slave conçoit les rapports avec le monde surnaturel. Mais Mickiewicz a conscience de cette antinomie, qui s'est manifestée dans le débat entre Schlegel, partisan de la communauté spirituelle universelle, et Schleiermacher, privilégiant l'indidualité du sentiment religieux. Peut-être Mickiewicz trouve-t-il une solution partielle au problème dans la méthode dialogique de Solger, chez qui la conscience individuelle est une manifestation de la conscience universelle (III, XXIV, 464-467).

Quoiqu'il en soit, la préexistence des âmes («idées innées » selon le langage des «écoles»), en germe dans le sein de la Divinité, constitue pour Mickiewicz l'une des idées les plus anciennes de la "philosophie primitive révélée » (III, III, 33-34) : l'anamnèse platonicienne, associée à la théosophie martiniste, est bien la toile de fond de la pensée religieuse de Mickiewicz. Ces intuitions sont en accord avec la théologie mystique paulinienne, puis celle d'Augustin, selon lesquelles tout a été créé par le Verbe et préexistait dans le Verbe avant d'être créé. Mickiewicz admet le dogme de l'incarnation de ce Verbe divin en Jésus, et reproche à Leroux sa mise en question de la divinité du Christ et de la résurrection (II, XXXII, 353). Pourtant, il approuverait sans doute l'idée de Leroux selon laquelle la divinité du Christ n'est pas exclusive : le Verbe, identifié au génie, s'incarne successivement dans différentes époques, lorsque «l'étincelle divine s'obscurcit» et «la chair opprime l'esprit» (III, V, 84).

15 «Dieu, le vrai Dieu, le Dieu incompréhensible et caché bien qu'éternellement manifesté, se communique à nous dans une Révélation éternelle et successive. C'est cette Révélation que j'étudie dans les religions antérieures et dans les philosophies positives» (Leroux, 1845, p. VII). 
C'est même ici l'idée centrale du messianisme mickiewiczien, qui mène le poète à reconnaître en Towiański le Messie attendu, et dont il aurait prédit la venue dans la troisième partie des Ä̈eux (la vision du prêtre Pierre sur le Sauveur portant le nom "quarante et quatre») et dans son improvisation à l'occasion de l'inauguration du cours du Collège de France (Weintraub, 1998b). Le sujet de l'adhésion de Mickiewicz à la secte de Towiański et ses rapports avec celui-ci constituent un sujet à part, assez bien étudié déjà, qu'il ne nous appartient pas de traiter ici (Górski, 1986; Hoffmann-Piotrowska, 2004, 2014, Fabianowski \& Hoffmanna 2005, 2014; Rutkowski, 1999; Sikora, 1967, 1984). Nous sommes d'accord avec les chercheurs (Kolodziej, Sikora, Walicki) qui constatent une continuité au sein de l'œuvre de Mickiewicz, et voient dans le towianisme une conséquence logique et un accomplissement des recherches antérieures du poète ${ }^{16}$. Si la présence du prophète lituanien dans les cours est plus implicite qu'explicite, cela n'amoindrit en rien son impact sur les idées de Mickiewicz. Et même si l'on tient compte de la dégradation à venir des rapports entre les deux hommes, le messianisme de Towiański n'en demeure pas moins la clef de voûte du quatrième cours, qui se transforme progressivement en séance de prêche prophétique - ce qui résultera en sa fermeture. Si Mickiewicz a une certaine idée de la religion universelle, c'est bien une religion messianique, qui unit en soi la révélation première et l'évolution du monde terrestre, la tradition primitive du Verbe et le progrès qui rythme ses manifestations successives.

\section{La révélation après la révélation}

Le messianisme n'apparaît pas par hasard dans l'Europe de la période romantique : il est le symptôme d'une crise et d'une espérance. La perte de l'indépendance par la Pologne a contribué à l'expansion de ce type de conception, mais celle-ci, comme on le sait, n'est pas une création exclusivement polonaise. D’origine juive, elle s'incorpore dans la tradition de la pensée occidentale à travers le christianisme et ses prolongements millénaristes, aussi bien religieux que sécularisés ${ }^{17}$. Le mouvement des idées au XIX ${ }^{\mathrm{e}}$ siècle en témoigne bien,

${ }^{16}$ De ce point de vue, il est possible d'établir une analogie entre l'expérience towianiste de Mickiewicz et l'expérience des Tables de Hugo à Jersey.

17 Le messianisme romantique polonais et ses rapports avec d'autres messianismes et millénarismes ont fait l'objet d'études détaillées, surtout d'Andrzej Walicki (1970, 1983). 
et la vague de despotisme éclairé en Europe, l'apparition de la question sociale et la crise de la religion institutionnalisée y sont pour beaucoup.

Pierre-Simon Ballanche exprime dans ses œuvres l'idée que l'humanité passe par des panlingénésies, des cycles expiatoires et initiatiques qui la font progresser vers l'accomplissement des destinées, et font évoluer les systèmes sociaux aussi bien que les idées religieuses qui les sous-tendent. La pensée de Ballanche est intéressante dans la mesure où, admettant l'existence d'une révélation primitive et d'une religion universelle qui la perpétue, elle réunit dans une même vision les «traditions générales ${ }^{18}$ qui en sont issues et le progrès qui les fait évoluer dans le temps. La religion universelle de Ballanche n'est pas la religion naturelle des déistes, mais celle des théosophes ${ }^{19}$, se rapprochant ainsi du catholicisme - une idée que Mickiewicz partage à coup sûr, d'autant plus qu'elle ne recule pas devant un dépassement du cadre formel de la religion catholique. Or, c'est bien ce que Mickiewicz annonce dans son cours dirigé contre l'Église officielle :

Il ne s'agit pourtant pas, qu'on le sache bien, ni de réformes, ni d'innovations, ni
de révolutions religieuses, mais on s'attend à une nouvelle manifestation de l'esprit
chrétien. Le papillon qui, au lever d'un soleil printanier, s'élève vers le ciel, n'est pas
une chrysalide réformée, révolue ou innovée; c'est toujours le même être, mais élevé
à une seconde puissance de vie ; c'est une chrysalide transfigurée. L'esprit chrétien
est prêt à sortir du sein de l'Église catholique : seulement le clergé officiel n'a pas
assez de lumière et de chaleur pour le faire éclore. / En vérité, la masse de lumière
et de chaleur nécessaire pour faire éclore le germe vital de toute une époque est si
immense, qu'elle ne peut sortir que du sein de Dieu, et qu'elle ne peut se manifester
sur la terre que par une nouvelle explosion du Verbe de Jésus-Christ (IV, VII, 130;
Mickiewicz, 1845b, p. 130).

Le passage cité, qui fait partie des nombreux fragments où Mickiewicz annonce l'avènement d'une nouvelle époque, accentue à travers une métaphore biologique l'aspect évolutif des changements qui sont sur le point d'advenir. Cependant, s'il y a une continuité entre la chrysalide et le papillon, une transfiguration sépare néanmoins les deux formes d'être : le poète a conscience du

18 Ballanche écrit dans ses Prolégomènes: «Dieu a voulu de plus que nous méritassions par la foi ; et j'entends ici la foi dans un sens étendu, planant au-dessus de toutes les religions, pour ne s'appliquer qu'à ce que j'appelle les traditions générales, la religion universelle du genre humain» (Ballanche, 1830, p. 327).

${ }^{19}$ Un curieux amalgame de ces éléments est fourni par le philosophe post-hégélien polonais Trentowski, qui formule une théosophie rejetant la révélation. Trentowski est à la fois admiré et critiqué par Mickiewicz (Walicki, 2006). 
fait que le «changement universel» attendu (IV, IX, 166-167) nécessite, comme toute nouvelle naissance, les troubles et les douleurs de l'enfantement :

Il y a une masse de lumière et de chaleur donnée pour chaque époque, c'est ce qui constitue l'époque : elle est épuisée; il en faut une nouvelle dose pour ranimer l'humanité et faire surgir une époque nouvelle. Qu'on ne se fasse pas illusion en croyant que l'humanité n'a plus qu'à marcher à petits pas pour s'avancer sans danger ni secousse, non! Dans les régions de la vie tout marche par des crises (IV, X, 204; Mickiewicz, 1845b, p. 204).

Les crises dont parle Mickiewicz évoquent de nouveau les épreuves initiatiques qui sont à la base de la conception ballanchienne. Leur attente s'inscrit aussi dans une perspective millénariste et apocalyptique, suivant laquelle les fléaux, les guerres et les persécutions doivent précéder le nouveau règne et la nouvelle création. Les prophéties sur le renouveau universel et le passage à une nouvelle ère, celle de l'Esprit, sont à l'heure du temps dans la première moitié du XIX ${ }^{\mathrm{e}}$ siècle - continuation d'une tradition joachimite héritée du Moyen-âge et transmise par Lessing dans son Éducation du genre humain $(1780)^{20}$. Ainsi, de Maistre dans les Soirées de Saint-Pétersbourg, ouvrage dicté par le même enthousiamse religieux inspiré des milieux illuministes et maçonniques qu'a connu Mickiewicz, annonce une nouvelle effusion de l'Esprit et une «troisième explosion du christianisme ", ce que le poète polonais ne manque pas de noter. De Maistre prévoyait par ailleurs la venue d'un génie et d'un révélateur qui apporterait une réunion de la religion et de la science (III, XXIV, 478). Celle-ci constitue un motif déjà présent chez Lessing, repris par Ballanche, Leroux ou Renan. Mickiewicz, quant à lui, tout antirationaliste qu'il était, constatait que " [l']humanité est appelée a monter plus haut vers le ciel, et à descendre plus bas dans les mystères de la nature » (IV, XIV, 65), d'accord avec la Naturphilosophie allemande alimentée par le néoplatonisme, la théosophie de Bœhme et de Saint-Martin.

Mais si de Maistre attendait la venue de la nouvelle ère du christianisme au sein du catholicisme, Mickiewicz, comme Leroux ou Ballanche, prend ses distances par rapport aux institutions religieuses, qui ont tendance à pétrifier ce qu'il y a de vivant et de vrai dans la religion. En néomontaniste, Mickiewicz

${ }^{20}$ Cette conception est reprise par de nombreux auteurs, qu'il est impossible de tous énumérer ici. Citons à titre d'exemple les saint-simoniens, Ballanche ou Leroux, et en Pologne, Cieszkowski et Krasiński. Henri de Lubac a consacré une étude détaillée à La postérité spirituelle de Joachim de Flore (1979, 1981), dont Mickiewicz fait lui aussi partie. 
constate que l'esprit de prophétie est absent de l'Église de Rome, qui n'est plus capable de recevoir l'inspiration de la région supérieure. Or, la nouvelle époque sera celle de la religion de l'amour et de l'Église de Jean opposée à celle de Pierre, suivant les idées des spiritualistes joachimites médiévaux et plus récemment de Schelling (II, XXXII [III, LXXIII, 352]). Cette idée de l'opposition entre les deux Églises : l'une intérieure et l'autre extérieure, l'une inspirée et l'autre formaliste, est déjà évoquée de façon discrète par Mickiewicz dans Le livre des pélerins polonais (Weintraub, 1998b), elle constitue aussi le thème du poème La vision de la nuit de Noël de Krasiński analysé par Mickiewicz durant son quatrième cours (IV, IV, 58-63). La vision présente le pélerinage de l'armée polonaise à Rome. On y assiste à l'apparition de Saint Jean représentant l'Église de l'avenir. Saint Pierre meurt dans son tombeau et la basilique s'écroule, mais son dôme est soutenu par l'armée des pélerins ${ }^{21}$. Mickiewicz commente la vision de la façon suivante:

Cette légion des pélerins ne représente pas seulement les légions polonaises, quoiqu'elle contienne la tradition spirituelle de leurs migrations mystérieuses; elle représente aussi cette innombrable légion des hommes qui cherchent l'Église de l'avenir. Tous ils se dirigent vers Rome, tous ils doivent passer par Rome, tous ils doivent entrer dans cette basilique; mais ils ne périront pas dans ses ruines, ils arrêteront cette coupole sur leurs armes. Ce ne seront pas les armes matérielles ni les armes des individus qui parviendront à la sauver, mais les esprits des nations. Les esprits des nations retiendront cette coupole près de s'écrouler. Ils ouvriront cette coupole à la lumière du ciel, pour qu'elle ressemble à ce panthéon dont elle est la copie ; pour qu'elle devienne de nouveau la basilique de l'univers, le panthéon, le pancosme et le pandème, le temple de tous les esprits ; pour qu'elle nous donne la clef de toutes les traditions et de toutes les philosophies ${ }^{22}$ (IV, IV, 62-63; Mickiewicz, 1845b, pp. 62-63).

Ce rêve millénariste, et surtout ses dernières lignes, n'est pas sans rappeler les utopies ou les projets de réforme globale comme celui formulé au XVII siècle dans les manifestes des Rose-Croix, qui aspirait à fondre la totalité du savoir

${ }^{21}$ Le texte de Krasiński diffère en fait clairement de l'interprétation de Mickiewicz: les nobles polonais y périssent sous la coupole écroulée, fidèles au vieux pape. Ce sacrifice précède l'avènement de l'époque nouvelle de Jean (Bagłajewski, 2011; Masłowski, 2010).

${ }^{22}$ Nous retrouvons une idée similaire dans l'ouvrage Du Christianisme et de son origine démocratique de Leroux, paru en 1848 : «L'effet inévitable sera, comme nous le disions toutà-l'heure, de créer dans tous les esprits un Panthéon plus vaste, où le passé tout entier sera admis et prendra place, mais où le christianisme n'occupera que la sienne ; et ce Panthéon, il n'est donné qu'à une Doctrine générale nouvelle de l'élever : ce sera son monument et son temple» (Leroux, 1848, p. 42). 
humain, des sciences et des arts, dans la synthèse d'un christianisme ésotérique et alchimique. Le romantisme est un creuset spirituel où les anciennes traditions viennent se fondre avec les nouvelles façons de sentir, avec l'idée du progrès et la philosophie de l'histoire, jetant une nouvelle lumière sur les destinées de l'humanité. Ce qui attire aussi l'attention dans le passage cité est le rôle joué par les « esprits des nations » dans la constitution de l'Église de l'avenir, ainsi que l'opposition de ces esprits aux efforts et aux armes des individus. Opposition surprenante dans la mesure où l'on sait que Mickiewicz est loin d'être pacifiste (il reproche cette attitude aux socialistes utopiques) et qu'il souligne l'importance de l'initiative individuelle inspirée par l'enthousiasme.

En fait, les propos de Mickiewicz sont plutôt complémentaires que contradictoires. Pour lui, «à l'heure qu'il est, ce sont des soldats qui nous offrent le vrai type du christianisme passé et futur ", une manière de vivre monacale, active et spontanée (IV, VIII, 152). En même temps, il s'agit d'un modèle de vie qui favorise le travail spirituel et l'ascèse, qui permet de «servir d'organe à la Divinité », de devenir un instrument du Verbe. Mais l'importance de la force morale et militaire ${ }^{23}$, ainsi que le culte du héros inspiré de Dieu, ne peut se manifester que dans un contexte collectif, chaque nation étant dotée d'une mission qui lui est propre. Trois nations sont élues du point de vue messianique : les Polonais, qui ont le plus souffert, eux-mêmes faisant partie du peuple slave qui a préservé son innocence et sa noblesse originelles ; les Français, qui peuvent fournir la force de frappe, le génie militaire et le dévouement ; et enfin les Juifs, peuple élu et exilé, toujours en attente de son Messie. Le culte de Napoléon et celui de l'Euvre de Dieu towianiste viennent se greffer sur cette triade. Il est intéressant d'observer que les figures du Messie souffrant, humilié, et du Messie glorieux et réssucité s'y rencontrent, la seconde - napoléonienne - étant conditionnée par la première - slave et polonaise. La foi dans la venue prochaine d'un Messie victorieux et vengeur a son origine dans l'expérience de la défaite, de la déception et du désespoir.

Cependant, chez Mickiewicz, ce rapport entre la situation historique et le renouveau politico-religieux (christianisation des relations internationales) s'inscrit dans une vision historiosophique qui, à l'image de celle de Ballanche, Leroux ou Quinet, suppose le développement et l'actualisation d'une révélation première par la voie du progrès. Chaque période apporte un perfection-

${ }^{23}$ C’est notamment ce dernier élément qui provoque son conflit avec Towiański, qui finira par faire rédiger une lettre de soumission au tsar en novembre 1844. 
nement par rapport à la précédente ${ }^{24}$. En effet, « Progrès » est l'un des mots sacramentels de l'époque pour Mickiewicz, qui l'attribue à Saint-Martin et Condorcet - l'un de ces mots qui naissent en dehors de l'Église officielle et autour desquels s'articulent les destinées du monde moderne. Il est impossible pour l'humanité de revenir sur ses pas, de faire chemin arrière. Chaque époque a besoin d'un idéal nouveau, adapté à ses exigences et récapitulant tous les idéaux antérieurs (IV, XI, 222). C'est ainsi que Mickiewicz, en citant Emerson, conçoit la figure de l' "homme éternel» (IV, XII, 243), homme complet qui porte en soi l'expérience de tous les siècles passés :

L'histoire doit cesser d'être un bouquin : il faut absolument sortir du bouquin. L'histoire doit marcher incarnée dans chaque homme juste et sage. Ne me citez plus les catalogues des livres que vous avez lus : faites-moi sentir de quel siècle vous êtes, combien de siècles vous avez vécu. Apportez-moi la preuve vivante de l'époque à laquelle vous appartenez : c'est d'après cela que je vous classerai. Je dois retrouver dans l'homme le passé tout entier; dans son enfance, l'âge d'or, l'arbre de la science du bien et du mal, l'expédition des Argonautes, la vocation d'Abraham, la construction du temple, et dans sa jeunesse la venue du Christ, le moyen-âge, la découverte de I’Amérique, etc., etc. Si vous n'avez pas fait encore votre croisade et votre révolution française, dépêchez-vous, car autrement vous ne pourrez pas suivre la génération actuelle (IV, XII, 236-237; Mickiewicz, 1845b, pp. 236-237).

Cette conception progressiste est bien ce qui sépare Mickiewicz des penseurs radicalement traditionalistes et conservateurs, et le rapproche de l'héritage révolutionnaire, orenté vers l'avenir et la modernité, mais tout en le laissant enraciné dans le passé. Car Mickiewicz aspire à une synthèse de la tradition et du progrès : il s'agit de faire sa croisade aussi bien que sa révolution. Et il faut se souvenir que les traditionalistes eux aussi ne sont pas déintéressés de l'avenir. Le Sénateur des Soirées de Saint-Pétersbourg de Joseph de Maistre annonce « je ne sais quelle grande unité vers laquelle nous marchons à grands pas» (Maistre, 1822, p. 324). Or, ceci s'accorde parfaitement avec le message prophétique de Mickiewicz: «Les temps sont passés où les nations disaient : Chacun chez soi, chacun pour soi. En quoi consisterait le progrès des nations si elles ne tendaient pas vers la construction d'une unité religieuse, politique et sociale? » (III, XXV, 503-504; Mickiewicz, 1845a, pp. 503-504). Mais alors que de Maistre envisage l'avenir à la lumière des principes légitimistes de l'ancien

24 Stanisław Pigoń définit la vision du monde des cours parisiens de Mickiewicz comme un "perfectionnisme spiritualiste universel », «spirytualistyczny perfekcjonizm powszechny » dans l'original (Walicki, 2006, p. 25). 
ordre, Mickiewicz n'hésite pas rompre avec eux au nom du progrès. Pour lui, comme le remarque Walicki en se référant à Max Weber, le pouvoir ne repose ni sur une institution traditionnelle quelconque, ni sur la raison, mais sur l'inspiration charismatique (Walicki, 2006).

$\mathrm{Du}$ fait de son attachement à la tradition nobilière et catholique polonaise, Mickiewicz pourrait être qualifié de « démocrate réactionnaire» (Weintraub, 1998a). Mais la primauté revient toujours chez lui à l' "Esprit qui souffle où il veut», qui anime les prophètes et guide les peuples. Et les questions économiques et sociales ne l'intéressent que dans la mesure où elles concernent la réalisation des desseins du Verbe divin. Si Mickiewicz se rapproche du socialisme en 1848, c'est parce qu'il est convaincu, comme il l'annonce alors au pape suivant certains témoignages ${ }^{25}$, que «l'Esprit Saint est dans les blouses du peuple parisien », celui-ci étant devenu instrument de la libération universelle. En traitant le problème de la propriété dans le troisième cours, il souligne pour une nouvelle fois le besoin d'un renouveau spirituel qui soit précéder les changements socio-politiques :

Je termine en répétant que la question de la propriété est liée à la question religieuse, et il est évident que l'époque arrive où elle doit être organisée d'une manière nouvelle. Cette époque coïncide avec l'attente générale des peuples. Le monde sent le besoin d'une effusion de lumière nouvelle qui pourrait éclairer nos rapports avec Dieu, avec la nature, qui pourrait nous faire saisir avec plus de vérité et avec plus de profondeur le lien qui unit Dieu à l'homme et à la nature. Ce n'est qu'après avoir reçu cette lumière qu'on pourra aussi éclaircir d'une manière nouvelle et efficace les rapports entre l'homme et la propriété (III, XIX, 405-406; Mickiewicz, 1845a, pp. 405-406).

L'intérêt de Mickiewicz pour la «question sociale», le sens de la propriété et la valeur des choses, permet d'établir des liens entre ses idées et celles des utopistes sociaux comme Saint-Simon, Fourier, Cabet ou Buchez. Cela est d'autant plus intéressant que tous les auteurs cités sont préoccuppés par la question religieuse : Saint-Simon annonce en 1825 son «nouveau christianisme», censé devenir «la religion générale, universelle et unique», visant «l'amélioration la plus rapide possible de l'existence morale et physique de la classe la plus pauvre» (Saint-Simon, 1825, pp. 21, 45). Parmi les saint-simoniens dissidents, on retrouve Pierre Leroux ainsi qu'Auguste Comte, le père du positivisme, qui fonde aussi sa "religion de l'humanité». Fourier réinterprète les dogmes chrétiens et veut construire le paradis sur terre en appliquant le principe de

${ }^{25}$ Il faut toutefois noter que l'authenticité de ces témoignages est discutable (Masłowski, 2010). 
l'attraction passionnée. Cabet voit dans le communisme le vrai christianisme, religion sans temples ni prêtres. Buchez fait concorder la lutte sociale et le catholicisme.

L'attitude de Mickiewicz par rapport aux utopistes demeure ambiguë. Dans ses réflexions sur La Comédie non divine de Krasiński, il associe les "idées panthéistiques » des saint-simoniens et des fouriéristes à l'idéologie révolutionnaire de Pancrace et au nouveau monde qu'elle aspire à créer, fondé sur la force brutale, la passion et l'intelligence (III, X, 182-199). Il méprise le pacifisme des saint-simoniens, des fouriéristes et des owénistes, estimant que l'agrandissement de la puissance morale et matérielle ne peut pas se faire sans guerre (IV, VII, 130-135). Mais en même temps il fait l'éloge des communautés slaves primitives en les comparant aux phalanstères fouriéristes (I, VII, 82-83). Et il indique en Buchez celui qui «a essayé le premier de démontrer par la logique la doctrine du salut universel » (IV, IV, 57; Mickiewicz, 1845b, p. 57). Buchez figure d'ailleurs, aux côtés de Chateaubriand, de Maistre, Hugo, Lamennais et Michelet, parmi ceux qui ont renforcé selon Mickiewicz l'action religieuse et appartiennent à l'«Église vivante » (IV, V, 72-73), opposée à l'Église officielle. Ils sont tous laïcs, «hommes de douleur» et «hommes d'action». C'est par eux, et non plus par les représentants formels de Dieu sur la terre, que l'inspiration divine passe dans le monde. L' "action» et le dévouement, la volonté de souffrir pour une cause, est ce qui constitue pour Mickiewicz le véritable sceau de la vérité. On ne trouvera pas celle-ci dans les raisonnements des systèmes philosophiques (IV, II, 15-18).

Mickiewicz est un ennemi déclaré de la philosophie d'école hégélienne et post-hégélienne. Il apprécie pourtant l'un de ses représentants, August Cieszkowski, pour avoir annoncé la fin de la philosophie scolastique et le commencement d'une nouvelle philosophie de l'esprit, de la vie et de l'action (III, XXIV, 457-459), ainsi qu'un autre penseur utopiste, Ludwik Królikowski, qui considère la pénitence et le sacrifice comme méthode d'élévation de l'âme à la vérité (III, XXIV, 467). Ces propos ne pouvaient que plaire à Mickiewicz, qui pose la souffrance et le sacrifice comme condition d'accès à la révélation. En se référant à l'exigence de la discipline intérieure en vigueur parmi les philosophes jusqu'à Pythagore, Mickiewicz oppose à la philosophie idéaliste allemande la philosophie nationale slave, spiritualiste et messianiste, basée sur une révélation progressive (III, I, 10). Or, la nécessité du sacrifice, idée mise en relief par Baader, est mentionnée par Mickiewicz comme la première caractéristique du messianisme (II, XXXII, 414-419). Walicki remarque les 
affinités des idées mickiewicziennes avec celles de Saint-Martin, de Maistre et Ballanche, parlant de la valeur expiatoire du sacrifice et de l'épreuve, ainsi que de la régénération par l'effusion du sang (Walicki, 2006). Mais il faut ajouter encore que la nécessité du sacrifice ne constitue pas seulement l'un des dogmes de la révélation primitive universelle, elle possède aussi une valeur épistémologique : seule une vie de prière qui ne recule pas devant l'effort et la douleur permet de trouver la vérité et de vivre conformément à elle, car la vérité est toujours incarnée et vécue :

Celui, le seul de tous, qui possédait la vérité, qui l'a réalisée, Jésus-Christ, est le seul réellement de tous les révélateurs qui n’a rien écrit. On devrait méditer ce fait, on devrait en conclure que l'homme qui a composé des livres cherche la vérité peut-être, mais ne l'a pas trouvée; car s'il l'avait trouvée, il l'aurait déjà réalisée dans sa personne et dans les institutions qu'il aurait fondées (IV, IX, 171; Mickiewicz, 1845b, p. 171).

Cette critique du rationalisme et plus généralement de l'intellectualisme est dirigée aussi bien contre les philosophes ${ }^{26}$ que contre les représentants de l'Église officielle:

La manie de faire des raisonnements stériles sur les questions religieuses et sociales, manie qui dénote en général la fin d'une époque, vous démontre pourquoi ni les hommes de l'église officielle, ni les hommes de la science officielle, que l'on appelle doctrinaires, n'ont plus aucune autorité ; et lorsqu'il n'y a plus d'autorité, alors commence la discussion (IV, IX, 165; Mickiewicz, 1845b, p. 165).

Pourtant, la seule question qui devrait nous intéresser est de nature philosophique: «D’où nous venons et où nous allons, c'est là la question, question qu' il faut résoudre ${ }^{27}$. Il ne suffit plus de dire aux hommes qu'ils viennent du néant, et qu'ils vont au ciel ou en enfer» (IV, VIII, 154-155; Mickiewicz, 1845b, pp. 154-155). La même pensée revient constamment chez Mickiewicz : ce ne sont pas les rationalistes matérialistes, ni les autorités religieuses en place qui nous fourniront la réponse:

D'où nous venons et où nous allons ? question qui tourmente l'église officielle et la philosophie, et ne laissera pas de repos à l'humanité, jusqu'à ce que la réponse se laisse apercevoir de manière à obtenir le consentement unanime des esprits faits pour la comprendre. [...] Sommons les philosophes spéculatifs, socialistes et économistes

26 «Notre seul adversaire, notre cher adversaire à nous autres, philosophes, c'est Mickiewicz. il [sic] nous est moins adverse, que correspondant et symétrique», écrit Michelet dans le « dossier polonais » des Légendes démocratiques du Nord (Weintraub, 1975, p. 55).

${ }^{27}$ Cette question, outre son caractère universel, trouve une expression privilégiée dans les Extraits de Théodote, texte représentatif de la gnose valentinienne. 
de nous dire d'une manière intelligible s'ils savent eux-mêmes et s'ils peuvent nous enseigner d'où nous venons et où nous allons (IV, IX, 158-159; Mickiewicz, 1845b, pp. 158-159).

Les idées du monde actuel, les mots sacramentels de l'époque, ont été forgés par les laïcs et en dehors de l'Église: « Progrès », comme nous l'avons déjà mentionné, par Saint-Martin et Condorcet, "Fraternité », par la Révolution, "Exaltation» par Leroux, "Intuition» par Cieszkowski et Emerson (IV, VII, 112-114). Selon Mickiewicz, c'est l'intuition et l'inspiration qui sont à l'origine de toute création artistique, de toute philosophie, des grandes actions ainsi que du dévouement militaire (IV, III, 30-44). Et ce ne sont qu'elles qui peuvent donner la réponse à la seule question qui compte. Or, ce qui distingue le christianisme, c'est précisément le caractère individuel de l'inspiration et de la vérité. Il consiste en l'imitation de Jésus-Christ, et non pas en l'adoption d'un système ou de lois établies (III, XIII, 248). Mais il s'agit bien d'une religion universelle, rassemblant les aspirations de toute l'humanité :

Les peuples ont souffert, les peuples se sont avancés sur le chemin de la croix, les peuples sont en possession des mystères et des espérances que l'Église officielle est incapable de partager, incapable de comprendre. C'est pourquoi elle nous a accusés de vouloir introduire une religion nouvelle, oubliant que le christianisme lui-même n'était pas une religion nouvelle, mais qu'il est venu expliquer et compléter pour toujours toutes les religions (IV, V, 80; Mickiewicz, 1845b, p. 80).

Mickiewicz exprime ici une conviction fondamentale, formulée déjà au $V^{e}$ siècle par Augustin dans ses Rétractations, et partagée par nombre des contemporains du poète (de Maistre, Lamennais, Eckstein, Ballanche, Leroux ou Comte), mais que chacun comprend d'une manière différente : il n'y a qu'une seule religion, l'humanité n'en a jamais eu d'autre. Issue d'une révélation première, elle existe au-delà des traditions et des confessions particulières et se perfectionne d'âge en âge. L'idée de la révélation est commune aux traditionalistes catholiques, à Ballanche et Leroux. Celle d'évolution progressiste à Ballanche, Leroux, Quinet, Saint-Simon et Comte, ainsi qu'à Lamennais. Les utopistes prévoient la venue d'une nouvelle époque et la réalisation prochaine du Royaume de Dieu sur terre. Tous ces éléments se croisent chez Mickiewicz, qui tente de s'élever par sa vision prophétique au dessus des visions du monde contraires mentionnées par Hoëne-Wroński dans le Prodrome du Messianisme (II, XXIX [III, LXX, 304-305]) et représentées dans la Comédie non divine de Krasiński 
(III, X, 182-199, 208-209) - du conservatisme monarchiste réactionnaire et du progressisme républicain révolutionnaire. En ceci elle est proche des conceptions de Ballanche, Leroux et Quinet ${ }^{28}$.

Mais Mickiewicz a poussé plus loin son antirationalisme et son intuitionnisme - méfiant sinon hostile à la civilisation occidentale, ne désirant voler d'elle que ce «feu sacré » et cette force autoritaire qui animaient l'armée napoléonienne, en lesquels il voyait un espoir de libération pour les peuples. La vision mickiewiczienne, orientée vers l'avenir, est aussi empreinte de nostalgie patriotique. L'utopie qu'il rêve est toujours celle de l'ancienne République nobilière polono-lituanienne, libre de lois écrites, où la liberté absolue rejoint l'enthousiasme spontané et le sens de la justice. Il doit se référer aux cieux dépeints par Swedenborg pour en donner une idée (III, XXV, 499). Mais elle se veut en même temps réaliste et pratique, comme l'était la religion slave. Sans doute ce réalisme et ce sens pratique ont-ils fait défaut à Mickiewicz, comme à ces rêveurs entrevoyant l'âge de l'Esprit, mais qui manquent de patience, évoqués par Lessing dans son Éducation du genre humain. Son messianisme, à la fois religieux et politique (en attente d'un Messie victorieux, à l'instar de celui du peuple d'Israël), mais critique vis-à-vis de l'Église officielle et des partis en place, le pousse à voir l'élu divin en un homme qui ne partagera pas ses opinions, si éloigné de cet «homme éternel » et providentiel voulu par le poète. "On ne devrait faire des vers qu'après avoir fait un miracle » : il s'agit là d'une pensée de Saint-Martin chère à Mickiewicz, et dont il a douloureusement éprouvé les conséquences. Alors qu'il donne ses cours au Collège de France, le «wieszcz» n'écrit presque plus, et gardera le silence jusqu'à sa mort, le miracle n'ayant pas eu lieu. Toutefois, parmi les chantres de la tradition vivante de la religion universelle, et parmi la " postérité spirituelle de Joachim de Flore» (Lubac, 2014), Mickiewicz ne cesse d'occuper une place de choix.

${ }^{28}$ En ce qui concerne le contexte philosophico-historique de l'idée de religion universelle, une étude à part devrait être faite sur le rapport entre Mickiewicz et ses amis du Collège de France, Michelet et Quinet - même si le trio a déjà fait l'objet de nombreuses études (Weintraub, 1975; Wodzyńska, 1976). Quinet, durant les deux dernières années des cours mickiewicziens, se rapproche du towianisme, et semble même près d'adhérer à la secte à un certain moment. Les différences entre Mickiewicz et Michelet vont progressivement s'accroître : après avoir constaté «Nous sommes aussi unis de sentiments qu’opposés de méthode» au début de l'année 1845, en mars de la même année, il écrit déjà : "nous différons cependant de méthode, de principe peut-être, essentiellement ». Michelet s'oppose avant tout au culte de l'individu élu messianique, qu'il s'agisse de Jésus ou de Napoléon (Weintraub, 1975, pp. 64-65). 


\section{BIBLIOGRAPHIE}

\section{Sources mickiewicziennes}

Mickiewicz, A. (1845a). L'Église officielle et le messianisme: Cours de littérature slave du Collège de France: Première partie: Littérature et philosophie. Paris: Au comptoir des imprimeurs-unis. Retrieved 1 July 2017, from https://books.google.pl/books?id=k5CYsWGShh8C\&printse $\mathrm{c}=$ frontcover\&hl $=\mathrm{pl} \# \mathrm{v}=$ onepage $\& \mathrm{q} \& \mathrm{f}=$ false

Mickiewicz, A. (1845b). L'Église et le Messie: Cours de littérature slave du Collège de France: Deuxième partie: Religion et politique. Paris: Au comptoir des imprimeurs-unis. Retrieved 1 July 2017, from https://books.google.pl/books?id=E7w9AAAAYAAJ\&printsec=frontco ver\&hl=pl\#v=onepage \&q\&f=false

Mickiewicz, A. (1849a). Les Slaves: Cours professé au Collège de France (Vols. 1-3). Paris: Au comptoir des imprimeurs-unis.

Mickiewicz, A. (1849b). Les Slaves: Cours professé au Collège de France (Vol. 1). Paris: Au comptoir des imprimeurs-unis. Retrieved 1 July 2017, from https://archive.org/details/ lesslavescoursp00mickgoog

Mickiewicz, A. (1997a). Dzieła: Wydanie Rocznicowe 1798-1998: Vols. 8-11. Literatura słowiańska). (J. Maślanka, Ed., L. Płoszewski, Trans.). Warszawa: Czytelnik.

Mickiewicz, A. (1997b). Prelekcje paryskie: Wybór. (M. Piwińska, Ed., L. Płoszewski, Trans.) (Vols. 1-2). Kraków: Universitas.

Mickiewicz, A. (1833). Livre des pélerins polonais, traduit du polonais d'Adam Mickiewicz par le comte Ch. de Montalembert, suivi d'un Hymne à la Pologne, par F. de La Mennais. Paris: E. Renduel. Retrieved 28 February 2017, from http://gallica.bnf.fr/ark:/12148/bpt6k991100k

\section{Autres sources}

Ballanche, P.-S. de (1830). Prolégomènes. In P.-S. Ballanche, Euvres de M. Ballanche (Vol. 3). Paris: Librairie J. Barbezat. Retrieved 28 February 2017, from http://gallica.bnf.fr/ ark:/12148/bpt6k114063q

Lamennais, F. de (1817). Essai sur l'indifférence en matière de religion (Vol. 1). Paris: TournachonMolin et H. Séguin. Retrieved 28 February 2017, from http://gallica.bnf.fr/ark:/12148/ bpt6k61216738

Leroux, P. (1845). De l'humanité, de son principe et de son avenir (Vol. 1). Paris: Perrotin. Retrieved 28 February 2017, from http://gallica.bnf.fr/ark:/12148/bpt6k454802b

Leroux, P. (1848). Du Christianisme et de son origine démocratique (Nouvelle edition). Boussac: imprimerie de P. Leroux. Retrieved 28 February 2017, from http://premierssocialismes. edel.univ-poitiers.fr/document/fd1630/viewer

Maistre, J. de (1822). Les soirées de Saint-Pétersbourg, ou Entretiens sur le gouvernement temporel de la providence; suivis d'un Traité sur les sacrifices (Vol. 2). Paris: Rusand. Retrieved 23 May 2017, from http://gallica.bnf.fr/ark:/12148/bpt6k56506w 
Miłosz, C. (1985). La Terre d'Ulro: Méditation sur l'espace et la religion. (Z. Bonowicz, Trans.). Paris: Albin Michel.

Saint-Simon Claude-Henri de, (1825), Nouveau christianisme - Dialogues entre un conservateur et un novateur. Retrieved 10 June 2017, from http://gallica.bnf.fr/ark:/12148/btvlb8626839k.

\section{Travaux critiques}

Bagłajewski, A. (2011). Mickiewiczowska interpretacja Legendy Krasińskiego w prelekcjach paryskich. In M. Kalinowska, J. Ławski, \& M. Bizior-Dombrowska (Eds.), Prelekcje paryskie Adama Mickiewicza wobec tradycji kultury polskiej i europejskiej: Próba nowego spojrzenia (pp. 163-179). Warszawa: Wydawnictwa Uniwersytetu Warszawskiego.

Fabianowski, A., \& Hoffmann-Piotrowska, E. (Eds.). (2005). Mickiewicz mistyczny. Warszawa: Wydawnictwa Uniwersytetu Warszawskiego.

Górski, K. (1986). Mickiewicz - Towiański. Warszawa: Państwowe Wydawnictwo Naukowe.

Hazard, P. (1935). La Crise de la conscience européenne (1680-1715). Paris: Boivin et Librairie générale française.

Hoffmann-Piotrowska, E. (2004). Mickiewicz-towiańczyk: Studium myśli. Warszawa: Wydział Polonistyki Uniwersytetu Warszawskiego.

Hoffmann-Piotrowska, E., (2014). „Święte awantury...”: orto- i heterodoksje Adama Mickiewicza. Warszawa: Wydział Polonistyki Uniwersytetu Warszawskiego.

Kalinowska, M., Ławski, J., \& Bizior-Dombrowska, M. (Eds.). (2011). Prelekcje paryskie Adama Mickiewicza wobec tradycji kultury polskiej i europejskiej: Próba nowego spojrzenia. Warszawa: Wydawnictwa Uniwersytetu Warszawskiego.

Kaźmierczyk, Z. (2012). Słowiańska psychomachia Mickiewicza. Gdańsk: Wydawnictwo Uniwersytetu Gdańskiego.

Kolodziej, L. (1966). Adam Mickiewicz: Au carrefour des romantismes européens, essai sur la pensée du poète. Aix-en-Provence: Éditions Ophrys.

Kubik, D. (2012). Mickiewiczowski projekt kultury słowiańskiej: Zarys problematyki dyskursu kulturoznawczego w prelekcjach paryskich. Zeszyty Naukowe Towarzystwa Doktorantów UJ. Nauki Humanistyczne, (4/1), 61-82.

Kuziak, M. (2007). O prelekcjach paryskich Adama Mickiewicza. Słupsk: Wydawnictwo Naukowe Akademii Pomorskiej.

Lagrée, J. (1991). La religion naturelle. Paris: Presses universitaires de France.

Linkner, T. (2014). W romantycznym kręgu słowiańskich wierzeń: Trentowski, Mickiewicz, Słowacki, Budzyński, Kraszewski. Gdańsk: Wydawnictwo Uniwersytetu Gdańskiego.

Lubac, H. de. (2014). La postérité spirituelle de Joachim de Flore: De Joachim à nos jours Euvres complètes XXVII-XVIII. (M. Sutton, Ed.). Paris: Les Éditions du Cerf.

Makowiecka, Z. (1968). Mickiewicz w Collège de France: Październik 1840 - maj 1844. Warszawa: Państwowy Instytut Wydawniczy.

Masłowski, M. (1998). Gest, symbol i rytuały polskiego teatru romantycznego. Warszawa: Wydaw. Naukowe PWN. 
Masłowski, M. (2002). La synthèse religieuse de Mickiewicz. In M. Masłowski \& F.-X. Coquin (Eds.), Le verbe et l'histoire: Mickiewicz, la France et l'Europe (pp. 150-165). Paris: Éditions de la Maison des sciences de l'homme.

Masłowski, M. (2010). Słowiański papież: Stosunek romantyków do Kościoła. Znak, (661), 71-87.

Piwińska, M. (1997). Dzieje kultury polskiej w prelekcjach paryskich. In A. Mickiewicz, Prelekcje paryskie: Wybór (Vol. 1, pp. 5-77). Kraków: Universitas.

Prussak, M. (2011). Problemy edytorskie wykładów Adama Mickiewicza w Collège de France. In M. Kalinowska, J. Ławski, \& M. Bizior-Dombrowska (Eds.), Prelekcje paryskie Adama Mickiewicza wobec tradycji kultury polskiej i europejskiej: Próba nowego spojrzenia (pp. 17-26). Warszawa: Wydawnictwa Uniwersytetu Warszawskiego.

Rutkowski, K. (1999). Braterstwo albo śmierć: Zabijanie Mickiewicza w Kole Sprawy Bożej. Gdańsk: Słowo / Obraz Terytoria.

Sikora, A. (1967). Posłannicy słowa: Hoene-Wroński, Towiański, Mickiewicz. Warszawa: Państwowe Wydawnictwo Naukowe.

Sikora, A. (1984). Towiański i rozterki romantyzmu. Warszawa: Wiedza Powszechna.

Stefanowska, Z. (1976). Próba zdrowego rozumu: Studia o Mickiewiczu. Warszawa: Państwowy Instytut Wydawniczy.

Stefanowska, Z. (2014). Mapa romantyzmu polskiego: Pisma z lat 1964-2007. Warszawa: Instytut Badań Literackich.

Walicki, A. (1970). Filozofia a mesjanizm: Studia z dziejów filozofii i myśli społeczno-religijnej romantyzmu polskiego. Warszawa: Państwowy Instytut Wydawniczy.

Walicki, A. (1983). Między filozofią, religią i polityką: Studia o myśli polskiej epoki romantyzmu. Warszawa: Państwowy Instytut Wydawniczy.

Walicki, A. (2002). La philosophie de l'Histoire d'Adam Mickiewicz. In M. Masłowski \& F.-X. Coquin (Eds.), Le verbe et l'histoire: Mickiewicz, la France et l'Europe (pp. 172-187). Paris: Éditions de la Maison des sciences de l'homme.

Walicki, A. (2006). Mesjanizm Adama Mickiewicza w perspektywie porównawczej. Warszawa: Instytut Badań Literackich PAN.

Walicki, A. (2009). Filozofia polskiego romantyzmu. Kraków: Universitas.

Weintraub, W. (1959). Literature as prophecy: Scholarship and martinist poetics in Mickiewicz's Parisian lectures. Gravenhage: Mouton and Co.

Weintraub, W. (1975). Profecja i profesura: Mickiewicz, Michelet i Quinet. Warszawa: Państwowy Instytut Wydawniczy.

Weintraub, W. (1998a). Mickiewicz - mistyczny polityk i inne studia o poecie. Warszawa: Instytut Badań Literackich PAN.

Weintraub, W. (1998b). Poeta i prorok: Rzecz o profetyzmie Mickiewicza. Warszawa: Biblioteka Narodowa.

Wodzyńska, M. (1976). Adam Mickiewicz i romantyczna filozofia historii w Collège de France. Warszawa: Państwowe Wydawnictwo Naukowe. 


\section{Prelekcje paryskie Adama Mickiewicza i idea religii uniwersalnej}

Pierwsza połowa XIX wieku przynosi we Francji rozwój wielu nurtów usiłujących znaleźć rozwiązanie problemów trawiących społeczeństwo w tym okresie (pauperyzacja, kryzys tradycyjnych wartości, ucisk ludów europejskich). Katoliccy tradycjonaliści, iluminiści, socjaliści utopijni i humanitaryści często formułują ideę uniwersalnej religii, wywodzącej się z pierwotnego objawienia i rozwijającej się w procesie dziejowym, lub też ideę nowej religii przyszłości. Celem artykułu jest usytuowanie w tym kontekście prelekcji paryskich Adama Mickiewicza oraz poglądów polskiego wieszcza na temat religii Słowian i mesjanizmu. Tekst podzielony jest na dwie części, odpowiadające dwóm kluczowych aspektom zagadnienia: pierwsza część mówi o szukaniu objawienia poprzedzającego objawienie judeochrześcijańskie, druga zaś dotyczy zapowiedzi nowego objawienia, stanowiącego dopełnienie tego ostatniego.

Słowa kluczowe: Adam Mickiewicz, prelekcje paryskie, Collège de France, religia uniwersalna, religia Słowian, mesjanizm, objawienie, XIX wiek

\section{Adam Mickiewicz's Paris Lectures and the Idea of Universal Religion}

The first half of nineteenth century in France brings the development of several currents of thought that are trying to find a solution to the problems affecting society in this period (pauperisation, crisis of traditional values, oppressed nations). Catholic traditionalists, Illuminati, utopian socialists and humanitarians often formulate the idea of a universal religion, derived from a primal revelation and evolving through the ages, or the idea of a new religion of the future. The aim of the article is to situate in that wider context Mickiewicz's Paris Lectures and the convictions of the Polish poet on Slavic religion and Messianism. The text is divided into two parts, corresponding to the key aspects of the topic: the first part talks about the search for a revelation preceding the Judeo-Christian revelation, and the second one concerns the announcement of a new revelation, which completes and fulfills the former one.

Keywords: Adam Mickiewicz, Paris Lectures, Collège de France, universal religion, Slavic religion, messianism, revelation, nineteenth century 


\section{Notka o autorze}

Tomasz Szymański (tomasz.szymanski@uwr.edu.pl) - doktor nauk humanistycznych, romanista, adiunkt w Instytucie Filologii Romańskiej na Uniwersytecie Wrocławskim. Autor wielu artykułów z zakresu literaturoznawstwa i historii idei w językach polskim i francuskim. Zainteresowania naukowe: literatura francuska (zwłaszcza romantyzmu), problematyka filozoficzna (antropologia, hermeneutyka, metafilozofia) i religijna (historia idei religijnych i duchowości, Zachodni ezoteryzm); obecny projekt: idea religii uniwersalnej we Francji w XIX wieku.

Tomasz Szymański, PhD (tomasz.szymanski@uwr.edu.pl) - Assistant Professor at the Institute of Romance Studies at the Faculty of Philology, University of Wrocław. Author of a number of articles on literature and history of ideas in Polish and French. Research interests: French literature (especially Romanticism), philosophy (anthropology, hermeneutics, metaphilosophy) and religion (history of doctrines and spiritualities, Western esotericism); current project: the idea of universal religion in nineteenth-century France. 